\title{
The effect of a flashing visual stimulus on the auditory continuity illusion
}

\author{
MaORI KobaYASHI \\ Rikkyo University, Niiza, Japan \\ and NTT Communication Science Laboratories, Atsugi, Japan \\ YoshIHISA OSADA \\ Rikkyo University, Niiza, Japan \\ AND \\ Makio Kashino \\ NTT Communication Science Laboratories, Atsugi, Japan
}

\begin{abstract}
The effect of a visual stimulus on the auditory continuity illusion was examined. Observers judged whether a tone that was repeatedly alternated with a band-pass noise was continuous or discontinuous. In most observers, a transient visual stimulus that was synchronized with the onset of the noise increased the limit of illusory continuity in terms of maximum noise duration and maximum tone level. The smaller the asynchrony between the noise onset and the visual stimulus onset, the larger the visual effect on this illusion. On the other hand, detection of a tone added to the noise was not enhanced by the visual stimulus. These results cannot be fully explained by the conventional theory that illusory continuity is created by the decomposition of peripheral excitation produced by the occluding sound.
\end{abstract}

The auditory continuity illusion is a compelling perceptual effect in which a sound (the inducee) interrupted momentarily by an extraneous sound (the inducer) is perceived as continuing through the inducer, even if the inducee is, in fact, physically absent during the presentation of the inducer (Houtgast, 1972; Miller \& Licklider, 1950; Warren, Obusek, \& Ackroff, 1972; see Warren, 1999, for a review). This phenomenon not only is a powerful illusion, but also represents an important ecological function of the auditory system that compensates for the effect of the masking by extraneous sounds often encountered in everyday situations. However, the mechanism underlying the auditory continuity illusion is not fully understood.

Some clues have been obtained concerning the processing stage at which the illusion is created. It has been shown that the continuity illusion involves frequency-selective processes. It occurs only when there is no decrease in excitation at the frequencies of the inducee during the presence of the inducer (Darwin, 2005; Houtgast, 1972; Warren et al., 1972). This masking potential rule indicates that peripheral filtering in the auditory system is involved in the process of creating the illusory continuity.

It has also been shown that the continuity illusion involves processes sensitive to binaural disparity. Kashino and Warren (1996) demonstrated that the illusion is inhibited when the interaural phase difference of the inducer and that of inducee are different and, as a consequence, the masking potential of the inducer is reduced due to the binaural masking level difference. This suggests that illusory continuity is produced after binaural convergence at the level of the brainstem nuclei. Darwin, Akeroyd, and Hukin (2002) also demonstrated the binaural contribution to the continuity illusion, using Huggins pitch.

There is also evidence that the continuity illusion involves processes that operate after the information from different frequency channels is integrated. Darwin (2005) examined the effect of simultaneous grouping of frequency components on the continuity illusion and demonstrated that judgments of auditory continuity are based on entire, simultaneously grouped objects, rather than solely determined by local criteria based on individual frequency channels.

Several studies have indicated that the process responsible for the auditory continuity illusion should operate before the perceptual attributes (e.g., loudness, pitch, etc.) of an auditory object are computed. For example, the apparent loudness of the inducer is reduced when continuity is perceived (McAdams, Botte, \& Drake, 1998; Warren, Bashford, Healy, \& Brubaker, 1994). Furthermore, continuity improves fundamental frequency (pitch) discrimination for unresolved complex tones (Plack \& White, 2000).

M. Kobayashi, maori@ais.riec.tohoku.ac.jp 
Some studies in which a forced choice task has been employed have shown that the continuity illusion can impair performance (Petkov, O'Connor, \& Sutter, 2003). This suggests that the continuity illusion is an automatic and compulsory process, free from the voluntary control of listeners. Another line of evidence suggesting that the continuity illusion is an automatic process has come from a mismatch negativity study, which showed that the illusion can occur outside the focus of attention (Micheyl et al., 2003).

The findings above suggest that the stage at which the continuity illusion is created is after binaural convergence and across-frequency integration but before the computation of perceptual attributes, such as loudness and pitch. Moreover, they suggest that the process is automatic and preattentive.

However, it is not fully clear how the continuity illusion is created in the auditory system. A cogent theory is that it results from the decomposition of the neural activity resulting from the inducer into the continuation of the inducee and a residue (Bregman, 1990; see Warren, 1999, for a review). This theory is consistent with both the masking potential rule and the loudness reduction of the inducer accompanying continuity (McAdams et al., 1998; Warren et al., 1994). However, this is not the only possible explanation for illusory continuity. Theoretically, continuity can be perceived unless a gap is explicitly represented in the auditory system. This does not necessarily involve the decomposition of the neural activity resulting from the inducer.

The present study was designed to provide additional empirical data that may help in the determination of where and how illusory auditory continuity is created within the auditory system. We examined whether the presentation of a visual stimulus can affect auditory continuity. One could think of various experimental settings in which to examine this. As was noted above, one of our motivations was to look for a factor influencing the continuity illusion that was not readily explained by the isomorphic view involving decomposition of neural activity for the inducer into the inducee and the residue. Our view is that a key factor governing perceived continuity is the perceptual interpretation of the onsets and offsets of sounds. Therefore, we decided to employ a transient, rather than a stationary, visual stimulus in the experiments. We expected that a transient visual stimulus would affect the detection and/or interpretation of sound onsets and offsets and, thus, alter the continuity illusion. In Experiment 1, we examined the effect of presenting a transient visual stimulus, synchronized with the onset of the inducer, on the limit of the perceiving of illusory continuity, when the limit was measured in terms of the maximum duration of inducee interruption. In Experiment 2, we examined the effect of the visual stimulus on the limit of the perceiving of illusory continuity when the limit was measured in terms of the maximum level of the inducee while maintaining the inducer level. The temporal tuning of the visual effect was also examined, because temporal synchrony is one of the critical factors affecting cross-modal interaction (Fujisaki, Shimojo, Kashino, \&
Nishida, 2004; Sekuler, Sekuler, \& Lau, 1997; Shams, Kamitani, \& Shimojo, 2002). In Experiment 3, we examined whether the visual stimulus affects the masking potential of the inducer.

\section{EXPERIMENT 1}

Experiment 1 was conducted to examine whether the presentation of a visual stimulus would affect the auditory continuity illusion at all. The observer's task was to judge whether the inducee appeared continuous at various inducer durations while the inducee duration was fixed. The continuity limit was defined as the longest inducer duration at which the inducee was judged to be continuous.

\section{Method}

Observers. Eleven observers (20-28 years of age) took part in this experiment. All had normal or corrected-to-normal vision and normal hearing (thresholds of $15 \mathrm{~dB} \mathrm{HL}$ or better at $125-8000 \mathrm{~Hz}$ ).

Apparatus and Stimuli. Testing was conducted in a darkened soundproof room. The visual stimulus was presented on a 17 -in. CRT monitor (Sony HMD-H200) positioned at eye level, approximately $57 \mathrm{~cm}$ from the observers' eyes. The auditory stimulus was delivered to both ears through headphones (Stax SR- $\Lambda$ PRO). An Apple Macintosh G5 computer was used to control the experiment and record responses.

The visual stimulus was a white ring (with a luminance of $108 \mathrm{~cd} / \mathrm{m}^{2}$ ) $5^{\circ}$ in diameter, $1.5^{\circ}$ in width. The ring was presented for $20 \mathrm{msec}$ on a black background (with a luminance of $0.2 \mathrm{~cd} / \mathrm{m}^{2}$; see Figure 1). The auditory stimulus consisted of two sounds that were presented in alternating fashion, as shown in Figure 1. The inducee was a $500-\mathrm{Hz}$ sinusoidal tone, and the inducer was a one-third-octave noise band centered at $500 \mathrm{~Hz}$. The inducer was always presented at $60 \mathrm{~dB}$ SPL, and the inducee was presented at $57 \mathrm{~dB}$ SPL. The duration of the inducee was fixed at $400 \mathrm{msec}$, and the initial duration of the inducer was $200 \mathrm{msec}$. To reduce switching transients, signals were gated on and off with a 10-msec raised cosine ramp. The ramps between successive sounds completely overlapped.

Procedure. Throughout a session, a fixation point was presented at the center of the monitor. The observers judged whether the inducee was continuous or not while they looked at a fixation point. There were two visual conditions. In the flash condition, the visual stimulus was presented at each onset of the inducer. In the no-flash condition, only a fixation point was presented. The continuity limits were measured using an up-down method. The inducer duration varied from trial to trial, depending on the observer's previous response: A discontinuous response resulted in a decrease of the inducer duration, and a continuous response resulted in an increase. The inducer duration was changed in 30 -msec steps, with the starting value randomly determined at the start of each session within the range of $170-230 \mathrm{msec}$. The continuity limit in a session was calculated as the mean of the last 8 of 10 staircase reversal points. Two sessions were conducted for each condition, and the mean of the continuity limits for the two sessions was taken as a continuity limit for the condition.

\section{Results and Discussion}

For 10 out of 11 observers, the continuity limits for the flash condition were longer than those for the no-flash condition. The continuity limits for the flash condition ( $M=235.6 \mathrm{msec}, S E=0.566)$ were longer than those for the no-flash condition $(M=215.3 \mathrm{msec}, S E=0.568)$ by $20.3 \mathrm{msec}$ across observers $[t(10)=2.24, p<.05]$. These results indicate that, overall, the presentation of the visual stimulus enhanced the continuity illusion. 


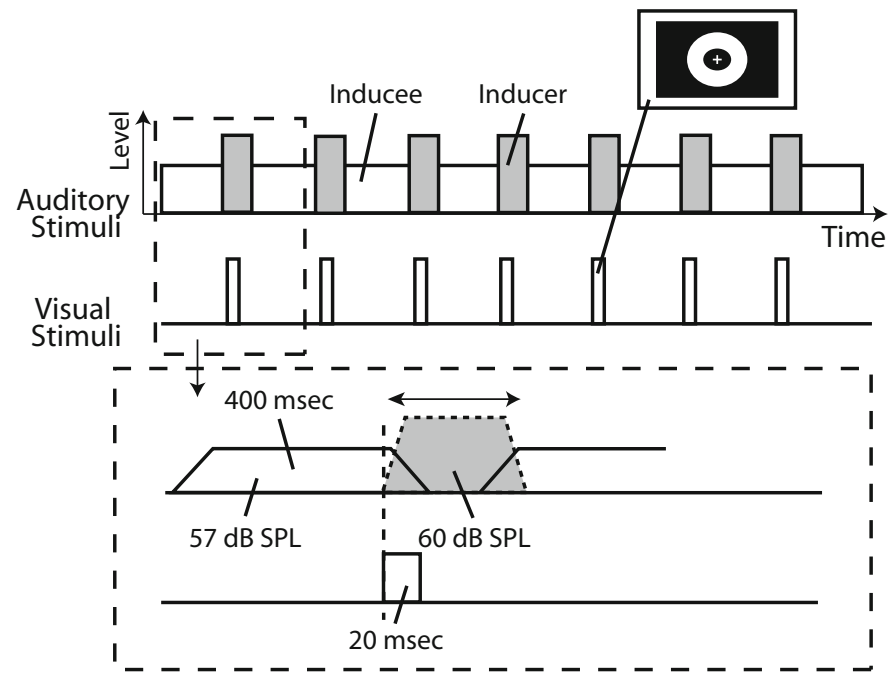

Figure 1. Stimuli used in Experiment 1. The visual stimulus was a white ring. The auditory stimuli employed for measuring the continuity limits consisted of a $500-\mathrm{Hz}$ sinusoidal inducee alternating with a $500-\mathrm{Hz}$, one-third-octave noise band inducer. The duration of the inducer varied from trial to trial, depending on the observer's previous response.

\section{EXPERIMENT 2}

To reinforce the finding of Experiment 1, the visual effect on the continuity illusion was evaluated differently. That is, the limit of the perceiving of illusory continuity was assessed in the domain of sound pressure level, a factor directly related to the masking potential rule. We also examined the effect of temporal synchrony between the visual stimulus and the inducer.

The observer's task was to judge whether the inducee appeared continuous at various inducee levels while the inducer level was fixed. The continuity limit was defined as the highest inducee level at which an observer judged the inducee as continuous.

\section{Method}

Observers. Eleven observers (21-34 years of age) took part in Experiment 2. None had participated in Experiment 1. Again, all had normal or corrected-to-normal vision and normal hearing.

Apparatus and Stimuli. The apparatus and visual stimulus were the same as those used in Experiment 1. The inducee duration was $400 \mathrm{msec}$, and the inducer duration was $200 \mathrm{msec}$, both fixed. To reduce switching transients, signals were gated on and off with a 10 -msec raised cosine ramp. The ramps between successive sounds completely overlapped, so that the entire sequence lasted 4,450 msec. The inducer was always presented at $60 \mathrm{~dB} \mathrm{SPL}$, whereas the inducee was presented in a range from 42 to $69 \mathrm{~dB}$ SPL on each trial.

Procedure. Throughout a session, a constant fixation point was presented at the center of the monitor. The observers judged whether the inducee was continuous or not while they looked at a fixation point. Seven conditions were tested: five stimulus onset asynchronies (SOAs) between the onset of the inducer and the onset of the visual stimulus $(-200,-100,0,+100,+200 \mathrm{msec}$, where negative values indicate that the inducer preceded the visual stimulus and positive values indicate that the inducer followed the visual stimulus), a random condition, and the no-flash condition. In the random condition, the SOA between a visual flash and each inducer was randomly chosen from seven values between -396 and $+396 \mathrm{msec}$ in a sequence, so that the timing of the visual flashes and the inducer levels were not correlated. The continuity limits were measured using a methodof-limits procedure. On the beginning trial of an ascending series of judgments, the inducee was presented at $42 \mathrm{~dB}$ SPL and increased regularly in steps of $3 \mathrm{~dB}$ in successive trials until the participant detected discontinuity in the inducee. For a descending series of trials, the inducee was first presented at $69 \mathrm{~dB}$ SPL and decreased in 3 -dB steps until the participant judged the inducee to be continuous. ${ }^{1}$ In each condition, the continuity measurement involved 12 series ( 6 ascending and 6 descending). The mean of all 12 stop points was taken as a continuity limit for the condition. The experiment lasted about $40 \mathrm{~min}$, with a pause halfway through the session.

\section{Results and Discussion}

Figure 2A shows the mean continuity limits for all 11 observers. The results differed somewhat among observers. For 9 observers, the continuity limits were highest in the 0 -msec condition (Figure 2B), whereas for the other two observers (M.U. and H.I.), the continuity limits were lowest in the 0 -msec condition (Figures $2 \mathrm{C}$ and 2D).

Observers M.U. and H.I., who showed the opposite tendency from the rest of the observers, also showed an SOA dependence, in the sense that the largest effect was obtained in the 0 -msec condition (it was simply in the opposite direction). This indicates that these 2 observers were not merely confused by the visual flashes when they judged continuity. Instead, they seem to have used a different perceptual strategy than did the rest of the observers.

We conducted a repeated measures ANOVA on the mean continuity limits for 9 observers, excluding the 2 outliers (Figure 2B), which revealed significant differences between the conditions $[F(6,48)=13.04, p<$ $.01] .{ }^{2}$ All pairwise comparisons, using the Tukey HSD test, revealed significant differences between the no-flash condition and the -200-, -100-, 0-, and +100-msec SOA conditions $(p<.01)$; between the random condition and the $-100-, 0-$, and $+100-\mathrm{msec}$ SOA conditions $(p<.01)$; 


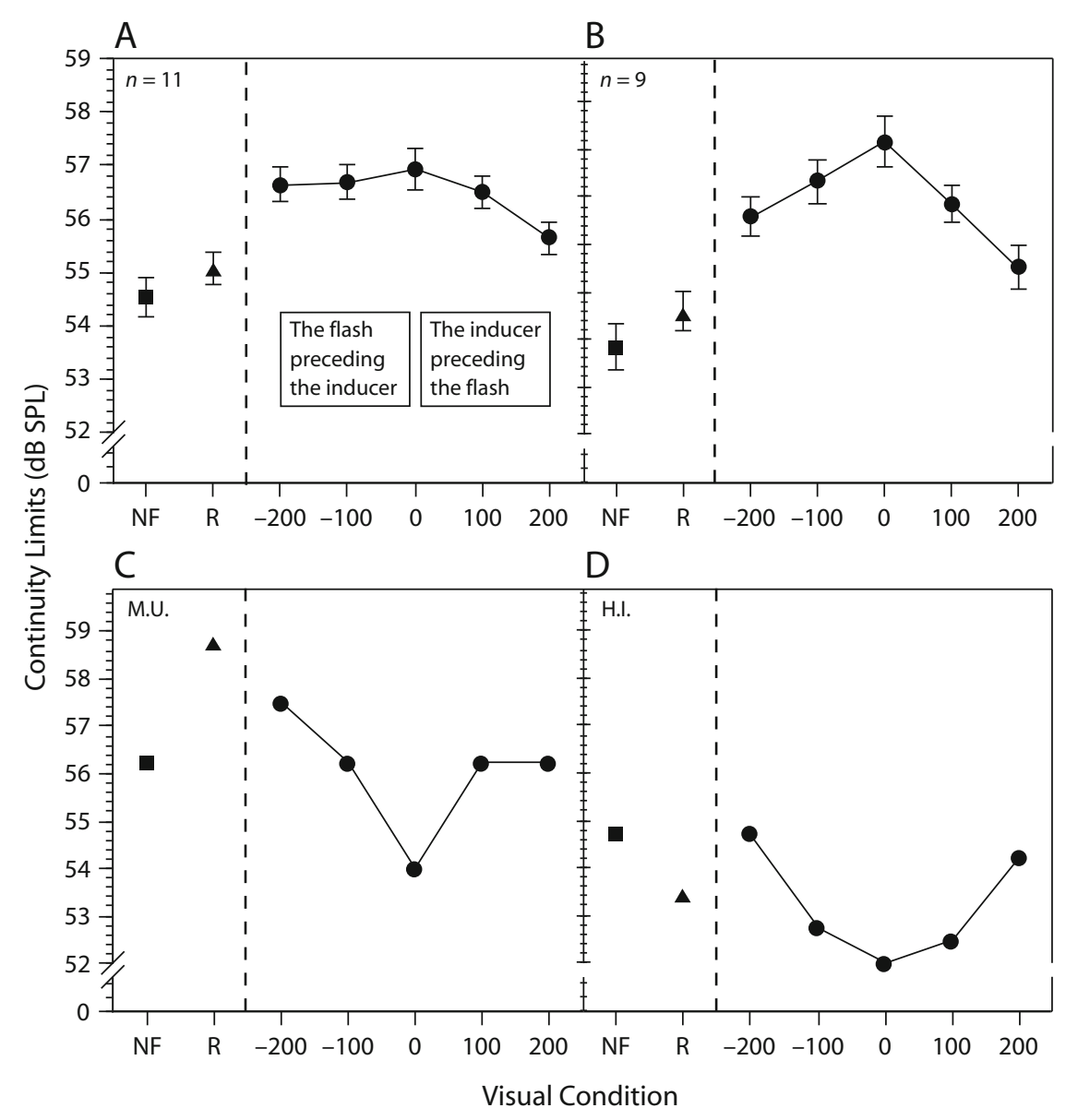

Figure 2. Continuity limits as a function of the seven conditions in Experiment 2 (NF, the no-flash condition; $R$, the random condition; the numbers are the stimulus onset asynchrony values, in milliseconds). (A) Means and standard errors for all the observers. (B) Means and standard errors for all the observers except M.U. and H.I. (C) Data for observer M.U. (D) Data for observer H.I.

between the -200 -msec condition and the random condition $(p<.05)$; and between the 0 -msec condition and the +200 -msec condition $(p<.01)$.

These results indicate, first, that the visual stimulus affects the continuity illusion, confirming the result of Experiment 1. Specifically, in most observers, the continuity illusion was enhanced when a visual stimulus was presented in synchrony with the onset of the inducer. The strength of the effect varied with the SOA between the inducer and the visual flash. Previous studies have reported that audiovisual synchrony is usually optimal for audiovisual interaction (Sekuler et al., 1997; Shams et al., 2002; Slutsky \& Recanzone, 2001). The SOA dependency of the visual effect on the auditory continuity illusion in this experiment is consistent with that reported in the previous studies (Sekuler et al., 1997; Shams et al., 2002). The visual stimulus had no significant effect on the continuity illusion in the random condition. It is therefore difficult to consider that the visual effect was due to mere distraction of the observers' attention for the task. It is more likely that the visual influence was a genuine perceptual effect.

\section{EXPERIMENT 3}

The masking potential of the inducer is known to be a crucial factor determining the continuity illusion (Houtgast, 1972; Warren et al., 1972). Previous research of audiovisual interaction has indicated that a visual stimulus can enhance auditory sensitivity in certain conditions. For example, Lovelace, Stein, and Wallace (2003) demonstrated that the detection of low-intensity sounds can be improved by the presentation of simultaneous but taskirrelevant light, using a stimulus paradigm and analyses derived from signal detection theory. Therefore, a possible explanation of the visual effect on the auditory continuity illusion demonstrated in Experiments 1 and 2 is that the presentation of the visual stimulus enhanced the masking potential of the inducer. 
To evaluate this possibility, we conducted Experiment 3, in which the effect of the visual flash on the simultaneous masking potential of the inducer was examined.

\section{Method}

Observers. Five observers (28-34 years of age) took part in Experiment 3 . They all had normal or corrected-to-normal vision and normal audition. Four out of the 5 had participated in Experiment 2 (O.K., O.S., K.R., and M.U.). It should be noted that M.U. was one who showed the opposite tendency in Experiment 2.

Apparatus and Stimuli. The apparatus was the same as that in Experiment 2. The visual stimulus was the same as that in Experiment 1 . The auditory stimuli consisted of a pure tone $(500 \mathrm{~Hz}$, $50 \mathrm{msec}$ ) as a target and a band-pass noise (one-third-octave band centered at $500 \mathrm{~Hz}, 200 \mathrm{msec}$ ) as a masker. The SOA between the onset of the masker and the onset of the target was randomized on each trial, in the range between 0 and $100 \mathrm{msec}$. The level of the masker was fixed at $60 \mathrm{~dB}$ SPL, and the level of the target was selected randomly from among five values $(-4,-2,0,+2$, and $+4 \mathrm{~dB})$, relative to each observer's approximate detection threshold, estimated in preliminary sessions.

Procedure. The method of constant stimuli was used with a twointerval forced choice procedure. The masker was presented in both intervals of a trial, and the target was presented randomly in either the first or the second interval. The task of the observers was to indicate the interval containing the target while they looked at the fixation point on the monitor. In each trial, the two stimulus intervals were separated by a silent interval of $300 \mathrm{msec}$. Each burst had a 10 -msec raised cosine rise and fall. In the flash condition, the visual stimulus was synchronized with the onset of the masker. In the noflash condition, only a fixation point was presented throughout the session. The observers took part in a practice session, which was followed by 10 experimental blocks. In the practice session, feedback was provided by a sound after each response, whereas no feedback was provided in the experimental blocks. Each block consisted of 50 trials in the no-flash condition and 50 trials in the flash condition. One block lasted about $12 \mathrm{~min}$. The total duration of this experiment was about $4 \mathrm{~h}$, including rest time.

\section{Results and Discussion}

Panels A-E of Figure 3 show the percentages of correct detection as a function of target level for the 5 observers. Psychometric functions were estimated using the maximum likelihood method. The level of the target corresponding to the $75 \%$ point on the psychometric function was defined as the threshold. Threshold values (in $\mathrm{dB}$ SPL) were, in the flash and no-flash conditions, respectively, 62.6 and 62.2 for S.S., 67.5 and 67.4 for O.K., 70.1 and 70.8 for M.U., 56.8 and 55.6 for O.S., and 68.1 and 67.4 for K.R. There was no significant effect of the visual condition $[t(4)=1.072$, n.s. $]$.

The results indicate that the visual stimulus did not affect simultaneous masking. Moreover, the results for M.U., who showed the opposite tendency from the majority of the observers in Experiment 2, were similar to those for the rest of the observers in this experiment. These results exclude the possibility that the visual effect on the auditory continuity illusion demonstrated in Experiments 1 and 2 is due to the change of masking potential of the inducer caused by the presentation of the visual stimulus.

\section{GENERAL DISCUSSION}

In Experiment 1, we examined the effect of presenting a transient visual stimulus, synchronized with the onset of the inducer, on the auditory continuity illusion. The results of Experiment 1 showed that presenting a transient visual stimulus affected the continuity illusion and that the effect was, in most listeners, in the direction that enhances illusory continuity. Next, we conducted Experiment 2 to reinforce the finding of Experiment 1, using a different measure - namely, the continuity limit in terms of sound

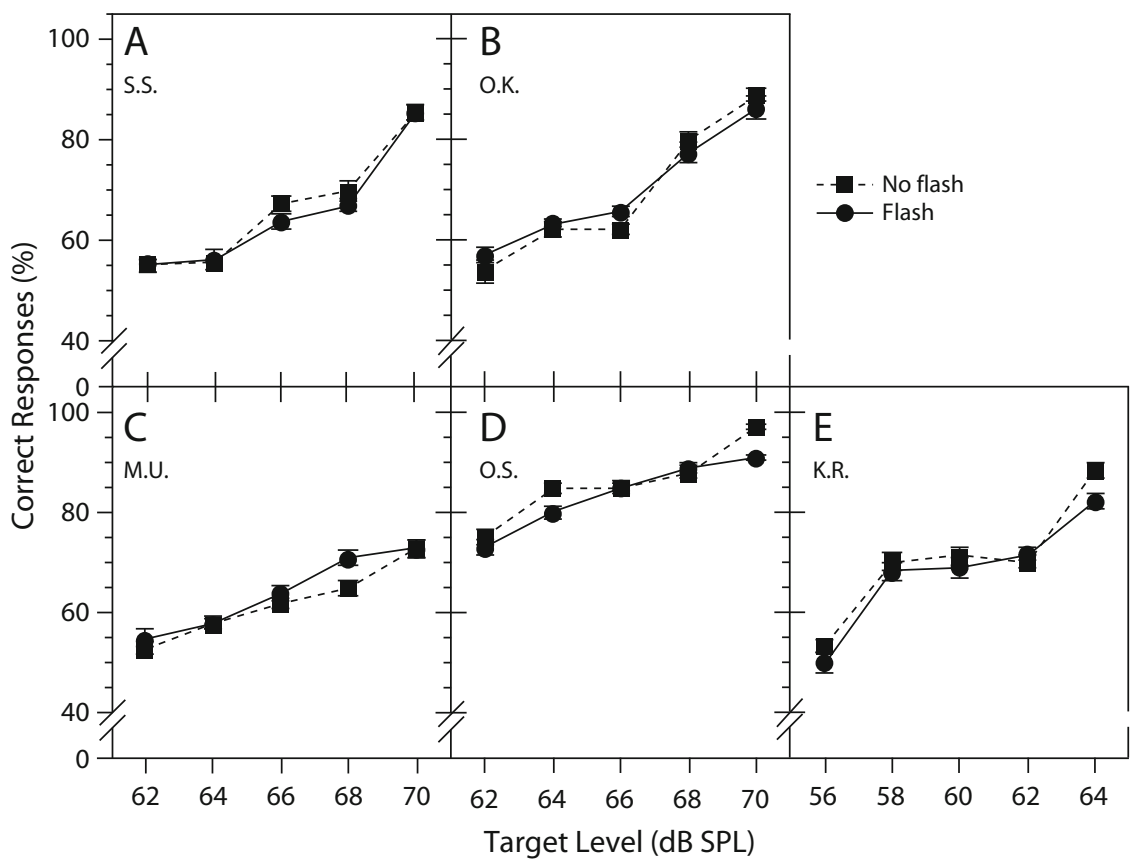

Figure 3. Percentages of correct responses as a function of the five tone-level conditions in Experiment 3. (A-E) Means and standard errors for each observer. 
level. We also examined the temporal tuning of the visual effect on the continuity illusion, because previous studies have indicated that the synchrony of signals from different modalities is among the critical factors determining crossmodal interaction. The results of Experiment 2 showed that the visual effect on the continuity illusion depended on the synchrony of the visual stimulus and the inducer, with the maximum effect occurring when the onsets of the visual stimulus and the inducer were synchronous. A possible explanation of the visual effect on the continuity illusion demonstrated in Experiments 1 and 2 is that the presentation of the visual stimulus enhanced the masking potential of the inducer. In Experiment 3, we examined whether the visual stimulus affects the masking potential of the inducer. The results showed that the visual stimulus did not affect auditory simultaneous masking.

The present findings provide an additional clue to the processing stage at which the continuity illusion is created. Previous studies have suggested that the stage is after binaural convergence and across-frequency integration but before the computation of perceptual attributes, such as loudness and pitch. According to the present finding of a visual effect on the auditory continuity illusion, the process of determining continuity appears to receive input from the visual system. The fact that no visual effect was found for auditory masking suggests that the site of the interaction should not be in the very early stages of auditory processing, which is consistent with previous findings. It is not possible at this point to specify the exact neural sites at which the audiovisual interaction in the auditory continuity illusion takes place. A possible site would be the auditory cortex, because neurophysiological studies have revealed that activation in the auditory cortex is modulated by visual stimuli (Calvert et al., 1997; Sams et al., 1991). Further research on the present effect, using different approaches such as functional imaging, may be promising.

The dissociation in the visual effect between the continuity illusion (Experiments 1 and 2) and masking (Experiment 3) brings up a novel point concerning the fundamental issue of how illusory continuity is created. The conventional theory is the old-plus-new heuristic, which assumes that the neural excitation evoked by the inducer is decomposed into the continuation of the inducee (an old event) and the residue (a new event) (Bregman, 1990; see Warren, 1999, for a review). This theory is supported by the masking potential rule and the loudness reduction of the inducer that accompanies the continuity illusion (Warren et al., 1994). However, the present study has identified a factor other than masking potential that affects the continuity illusion - namely, the presentation of a transient visual stimulus synchronized with the onset of the inducer. This suggests that the old-plus-new process, if it exists, cannot explain all of the processes involved in the formation of illusory continuity.

Here, we propose an alternative theory: Continuity of an object will be perceived unless the discontinuity of the object is explicitly represented in the auditory system. This is not contradictory to the masking potential rule, because the rule ensures that the discontinuity of the inducee will not be detected in relatively early stages of auditory processing. Our point is that masking of discontinuity is not the only way to hide it. Discontinuity may not be represented explicitly if attention is not directed to it. A possible explanation for the results of Experiments 1 and 2, according to this theory, is that the transient visual stimulus presented temporally close to the onset of the inducee captured attention, which otherwise would have been directed to the discontinuity of the inducee, resulting in the enhancement of the continuity illusion. This explanation is supported by an informal observation that the presentation of a prolonged visual stimulus synchronized with the entire sequence of the inducee had no effect on the continuity illusion. Theoretically, in order to perceive (illusory) continuity, it is not necessary for neural activity to be produced in the auditory system corresponding to the continuation of the object. Rather, the perceived continuity is a matter of interpretation of the onsets and offsets in terms of auditory objects. This idea is consistent with recent findings on the illusory recouplings of onsets and terminations of glide tone components (Nakajima et al., 2000 ) and the illusory short tone in stimulus patterns consisting of two partly overlapping glides (Remijn \& Nakajima, 2005). The performance of the observers who showed the opposite tendency in Experiments 1 and 2 can be explained if we assume that they bound the transient visual stimulus not to the onset of the inducer, as the majority of observers did, but to the offset of the inducee.

It may seem somewhat counterintuitive that continuity is enhanced when attention is not directed to the inducee. However, this is consistent with the finding by Micheyl et al. (2003) that the continuity illusion can occur outside the focus of attention. This may have an important functional role in the real world, where many auditory events often occur independently. Passive attention would be captured by the onset of a new event when one is listening to another event, but the representation for the continuing event should be maintained. The mechanism demonstrated in the present study may contribute to such maintenance of representation and to stable and efficient perception, in addition to the compensation of masking.

A possible criticism of the present study would be that Experiments 1 and 2 employed subjective measures of the continuity illusions and, therefore, were not free from observer response bias. Completely excluding the response bias explanations may require further experiments employing some objective (performance) measures (Kluender \& Jenison, 1992; Lyzenga, Carlyon, \& Moore, 2005; Petkov et al., 2003). At this point, we just mention an observation against the response bias explanation: Some observers reported after the experiments that their prediction was that the flash should make the tone (the inducee) less continuous, but their actual performance was the exact opposite.

Finally, we will make a point in the context of audiovisual interaction research. The present study is, to our knowledge, the first demonstrating a visual effect on auditory perceptual organization. Although a number of audiovisual interaction phenomena have been reported (Driver, 1996; Howard \& Templeton, 1966; McGurk \& MacDonald, 1976; Recanzone, 2003; Shams et al., 2002), it is difficult to find audiovisual phenomena at the level of perceptual 
organization, except for the stream-bounce effect reported by Sekuler et al. (1997). They demonstrated that the perception of two visually identical objects moving across each other is bistable (streaming or bouncing) but that it is biased in favor of bouncing motion when a brief sound is presented at the moment that the visual objects coincide. The mechanism underlying this phenomenon is not understood. Watanabe and Shimojo (1998) suggested that the stream-bounce effect occurs because the attentive tracking of the moving objects is disrupted by the transient auditory signal. This theory, however, would be somewhat contradictory to the present results, in which perceived continuity in a modality was enhanced by a transient signal from another modality. Further analysis of similarities and differences in these effects would reveal general principles of sensory information processing in the brain. It might also be fruitful to examine such topics as the effect of the saliency of the visual stimulus, the manipulation of an extraneous stimulus (e.g., auditory, tactile, or even pure expectation of a stimulus), and the manipulation of voluntary attention.

\section{AUTHOR NOTE}

The authors gratefully thank Yoshitaka Nakajima, Chris Darwin, and an anonymous reviewer for constructive comments on an earlier version of the manuscript and Eric W. Healy for improvement of the manuscript. Correspondence concerning this article should be addressed to M. Kobayashi, Research Institute of Technical Communication, Tohoku University, 2-1-1, Katahira, Aoba, Sendai, Miyagi, 980-8577 Japan (e-mail: maori@ais.riec.tohoku.ac.jp), or M. Kashino, NTT Communication Science Laboratories, NTT Corporation, 3-1, Morinosato-Wakamiya, Atsugi, Kanagawa, 243-0198 Japan (e-mail: kashino@avg.brl.ntt.co.jp).

\section{REFERENCES}

Bregman, A. S. (1990). Auditory scene analysis: The perceptual organization of sound. Cambridge, MA: MIT Press, Bradford Books.

Calvert, G. A., Bullmore, E. T., Brammer, M. J., Campbell, R., Williams, S. C., McGuire, P. K., ET AL. (1997). Activation of auditory cortex during silent lipreading. Science, 276, 593-596.

DARWIN, C. J. (2005). Simultaneous grouping and auditory continuity. Perception \& Psychophysics, 67, 1384-1390.

Darwin, C. J., Akeroyd, M. A., \& Hukin, R. W. (2002). Binaural factors in auditory continuity. In R. Nakatsu \& H. Kawahara (Eds.), Proceedings of the 8th International Conference on Auditory Display (pp. 259-262). Kyoto: ATR.

DrIVER, J. (1996). Enhancement of selective listening by illusory mislocation of speech sounds due to lip-reading. Nature, 381, 66-68.

Fujisaki, W., Shimojo, S., Kashino, M., \& Nishida, S. (2004). Recalibration of audiovisual simultaneity. Nature Neuroscience, $\mathbf{7}$, 773-778.

Houtgast, T. (1972). Psychophysical evidence for lateral inhibition in hearing. Journal of the Acoustical Society of America, 51, 18851894.

HowARD, I. P., \& TEMPLETON, W. B. (1966). Human spatial orientation. London: Wiley.

Kashino, M., \& Warren, R. M. (1996). Binaural release from temporal induction. Perception \& Psychophysics, 58, 899-905.

KLuENDER, K. R., \& Jenison, R. L. (1992). Effects of glide slope, noise intensity, and noise duration on the extrapolation of FM glides through noise. Perception \& Psychophysics, 51, 231-238.

Lovelace, C. T., Stein, B. E., \& Wallace, M. T. (2003). An irrelevant light enhances auditory detection in humans: A psychophysical analysis of multisensory integration in stimulus detection. Cognitive Brain Research, 17, 447-453.

Lyzenga, J., Carlyon, R. P., \& Moore, B. C. J. (2005). Dynamic as- pects of the continuity illusion: Perception of level and of the depth, rate, and phase of modulation. Hearing Research, 210, 30-41.

McAdams, S., Botte, M. C., \& Drake, C. (1998). Auditory continuity and loudness computation. Journal of the Acoustical Society of America, 103, 1580-1591.

MCGURK, H., \& MACDONALD, J. (1976). Hearing lips and seeing voices. Nature, 264, 746-748.

Micheyl, C., Carlyon, R. P., Shtyrov, Y., Hauk, O., Dodson, T., \& Pulvermüller, F. (2003). The neurophysiological basis of the auditory continuity illusion: A mismatch negativity study. Journal of Cognitive Neuroscience, $\mathbf{1 5}, \mathbf{7 4 7 - 7 5 8 .}$

Miller, G. A., \& LickLIDER, J. C. R. (1950). The intelligibility of interrupted speech. Journal of the Acoustical Society of America, 22, 167-173.

Nakajima, Y., Sasaki, T., Kanafuka, K., Miyamoto, A., Remijn, G., \& TEN Hoopen, G. (2000). Illusory recouplings of onsets and terminations of glide tone components. Perception \& Psychophysics, 62, 1413-1425.

Petkov, C. I., O’Connor, K. N., \& Sutter, M. L. (2003). Illusory sound perception in macaque monkeys. Journal of Neuroscience, $\mathbf{2 3}$, 9155-9161.

Plack, C. J., \& White, L. J. (2000). Perceived continuity and pitch perception. Journal of the Acoustical Society of America, 108, 1162-1169.

ReCAnzone, G. H. (2003). Auditory influences on visual temporal rate perception. Journal of Neurophysiology, 89, 1078-1093.

Remijn, G. B., \& NAKajIma, Y. (2005). The perceptual integration of auditory stimulus edges: An illusory short tone in stimulus patterns consisting of two partly overlapping glides. Journal of Experimental Psychology: Human Perception \& Performance, 31, 183-192.

Sams, M., Aulanko, R., Hamalainen, M., Hari, R., Lounasmaa, O. V., Lu, S. T., \& Simola, J. (1991). Seeing speech: Visual information from lip movements modifies activity in the human auditory cortex. Neuroscience Letters, 127, 141-145.

Sekuler, R., Sekuler, A. B., \& Lau, R. (1997). Sound alters visual motion perception. Nature, $\mathbf{3 8 5}, 308$.

Shams, L., Kamitani, Y., \& Shimojo, S. (2002). Visual illusion induced by sound. Cognitive Brain Research, 14, 147-152.

Slutsky, D. A., \& Recanzone, G. H. (2001). Temporal and spatial dependency of the ventriloquism effect. NeuroReport, 12, 7-10.

WARREN, R. M. (1999). Auditory perception: A new analysis and synthesis. Cambridge: Cambridge University Press.

Warren, R. M., Bashford, J. A., JR., Healy, E. W., \& Brubaker, B. S. (1994). Auditory induction: Reciprocal changes in alternating sounds. Perception \& Psychophysics, 55, 313-322.

Warren, R. M., Obusek, C. J., \& Ackroff, J. M. (1972). Auditory induction: Perceptual synthesis of absent sounds. Science, 176, $1149-1151$.

Watanabe, K., \& Shimojo, S. (1998). Attentional modulation in perception of visual motion events. Perception, 27, 1041-1054.

\section{NOTES}

1. Although it is not ideal to fix the starting points of the ascending and descending series, this is not critical in this experiment, given the following. First, in this experiment, a fresh noise having a certain amount of intrinsic level fluctuation was used as an inducer in each trial. Second, the initial sound level was well below or above the threshold. Third, perceptual changes around the threshold were not subtle, and the listeners did not have to rely on the number of trials from the start to make their judgments.

2. When the repeated measures ANOVA on the mean continuity limits includes all the observers, the differences between the conditions remain significant $[F(6,60)=5.689, p<.01]$, and the Tukey HSD test revealed significant differences between the no-flash condition and the -200 $-100-, 0-$, and +100 -msec SOA conditions and between the 0 -msec condition and the random condition.

(Manuscript received April 18, 2006; revision accepted for publication August 1, 2006.) 